\title{
Development of biologic coronary artery bypass grafting in a rabbit model: Revival of a classic concept with modern biotechnology
}

\author{
Koji Ueyama, MDa \\ Gao Bing, MD \\ Yasuhiko Tabata, $\mathrm{PhD}^{\mathrm{b}}$ \\ Makoto Ozeki, BS ${ }^{\text {b }}$ \\ Kazuhiko Doi, MDa \\ Kazunobu Nishimura, MD, PhD ${ }^{\mathrm{a}}$ \\ Hisayoshi Suma, MD, $\mathrm{PhD}^{\mathrm{c}}$ \\ Masashi Komeda, MD, PhDa
}

\begin{abstract}
From the Department of Cardiovascular Surgery, the Graduate School of Medicine, ${ }^{\text {a }}$ and the Institute for Frontier Medical Sciences, ${ }^{\text {b }}$ Kyoto University, Kyoto, Japan, and Hayama Heart Center, ${ }^{c}$ Kanagawa, Japan.

Read at the Eighty-third Annual Meeting of The American Association for Thoracic size was reduced to a greater extent in group FG than in groups $\mathrm{F}, \mathrm{G}$, and $\mathrm{N}(10 \%$ $\pm 3 \%, 16 \% \pm 5 \%, 19 \% \pm 7 \%, 23 \% \pm 2 \%$, respectively, group $\mathrm{F} P=.04$, groups $\mathrm{G}$ and $\mathrm{N} P<.01$ ). The number of arterioles 20 to $100 \mu \mathrm{m}$ in diameter was increased to a greater extent in group FG than in groups F, G, and N (23 \pm 5 arterioles $/ \mathrm{mm}^{2}$, $14 \pm 3$ arterioles $/ \mathrm{mm}^{2}, 10 \pm 1$ arterioles $/ \mathrm{mm}^{2}, 4 \pm 2$ arterioles $/ \mathrm{mm}^{2}$, respectively), with the differences being significant.
\end{abstract} Surgery, Boston, Mass, May 4-7, 2003.

Received for publication May 19, 2003; revisions requested Aug 8, 2003; revisions received Aug 11, 2003; accepted for publication Sept 17, 2003.

Address for reprints: Masashi Komeda, $\mathrm{MD}, \mathrm{PhD}$, Professor of Cardiovascular Surgery, Graduate School of Medicine, Kyoto University, 54 Shogoin, Sakyo-Ku, Kyoto, Japan PO: 606-8507 (E-mail: masakom@ kuhp.kyoto-u.ac.jp).

J Thorac Cardiovasc Surg 2004;127: $1608-15$

$0022-5223 / \$ 30.00$

Copyright $(92004$ by The American Association for Thoracic Surgery

doi:10.1016/j.jtcvs.2003.08.043

Conclusions: These results show that bypass from the gastroepiploic artery to coronary arteries can be achieved without surgical anastomosis through slow release of basic fibroblast growth factor in this rabbit acute myocardial infarction model. This new revascularization concept, biologic coronary artery bypass grafting, could be applicable for revascularizing many tiny coronary vessels in patients who are difficult to treat with conventional surgery or catheter intervention.

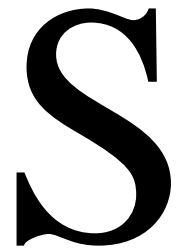

o-called therapeutic angiogenesis was developed as an alternative to conventional treatments, such as percutaneous coronary intervention and coronary artery bypass grafting $(\mathrm{CABG})^{1}$ for advanced coronary artery disease. Many experimental studies have demonstrated excellent results. ${ }^{2-7}$ Several clinical studies with therapeutic angiogenesis in patients undergoing CABG have shown improvement in myocar-
Objective: We have developed a technique for biologic coronary artery bypass grafting, which is a revival of a classic concept with modern biotechnology.

Methods: Acute myocardial infarction was created by ligating the major branch of the circumflex artery in rabbits. Animals were divided into four groups: a nontreated group (group N), a group in which omentum was used to wrap the infarcted area (group G), a group in which a gelatin hydrogel sheet incorporating $100 \mu \mathrm{g}$ basic fibroblast growth factor was placed over the infarcted area (group F), and a group in which the infarcted area was similarly treated with basic fibroblast growth factor followed by omental wrapping (group FG). Cardiac function was subsequently assessed by echocardiography. Postmortem angiography through the gastroepiploic artery was done in groups G and FG. Infarct size and arteriolar density were evaluated.

Results: Group FG showed a better fractional area change than did the other groups (group N $P<.001$, group G $P=.002$, group F $P<.001$ ). Angiography revealed that communication from the gastroepiploic artery to the coronary artery was created through a rich bed of neovascularization in all 7 animals of group FG, whereas poor collaterals were recognized in only 2 of 7 animals in group G. Infarct dial perfusion according to late nuclear perfusion scans. ${ }^{8,9}$ 
If the donor coronary artery from which new vessels sprouted by angiogenetic therapy was too far from the ischemic area, however, or if it was too small, diseased, or stenosed, then blood flow through the neovasculature might not be enough to supply the ischemic territory. The efficacy of current forms of therapeutic angiogenesis may not be sufficient for such types of coronary artery territory.

In 1936, O'Shaughnessy ${ }^{10}$ introduced a technique, omentopexy, in which the greater omentum was brought through the left diaphragm and wrapped around the ischemic heart. In 1950, Vineberg and Miller introduced internal thoracic artery implantation ${ }^{11}$ by modifying the omentopexy technique into a free omental graft operation. ${ }^{12}$ Although these classic procedures for treatment of angina pectoris required a long time for graft-coronary communication to mature sufficiently, some experimental studies have demonstrated the connection between omental vessels and the coronary artery by angiography, and some clinical reports have described a good outcome after pedicled or free omental grafting. ${ }^{3-16}$

We previously reported that use of a gelatin sheet incorporating basic fibroblast growth factor (bFGF) can offset sternal ischemia and accelerate sternal healing after harvesting of bilateral internal thoracic arteries in not only healthy but also diabetic rats. ${ }^{17,18} \mathrm{We}$ also reported that prevascularization with gelatin microspheres containing this angiogenic factor can enhance the benefits of cardiomyocyte transplantation. ${ }^{19}$ On the basis of these observations, we considered a new strategy for revascularization of severely diseased and tiny coronary arteries: (1) use of a healthy large-bored donor artery, such as the gastroepiploic artery (GEA), located near the ischemic area and (2) stimulation of angiogenesis from this donor artery soon after administration of an angiogenic factor. In other words, the strategy is a revival of the classic method, except that modern biotechnology is used (biologic coronary artery bypass grafting, or Bio-CABG). The purpose of this study was to demonstrate the feasibility of creating vascular communication between tiny coronary arteries and an extracardiac artery without surgical anastomosis by suitable placement of the artery and use of an angiogenic factor.

\section{Material and Methods}

\section{Preparation of Gelatin Hydrogel Sheet Incorporating bFGF}

Gelatin with an isoelectric point of 4.9 was isolated from bovine bone collagen by an alkaline process with calcium hydroxide (Nitta Gelatin Co, Osaka, Japan). The weight-average molecular weight of the gelatin was 99,000 d when measured by gel filtration chromatography relative to a standard polyethylene glycol sample. Human recombinant bFGF with an isoelectric point of 9.6 was obtained from Kaken Pharmaceutical Co (Tokyo, Japan). Gelatin hydrogel sheets were prepared as described previously elsewhere. ${ }^{17,20}$ The sheets were freeze-dried and then impregnated with an aqueous solution containing $100 \mu \mathrm{g}$ bFGF to obtain gelatin hydrogels with incorporated bFGF. The prepared hydrogel sheets were $5 \times 5 \mathrm{~mm}$ square and $0.7 \mathrm{~mm}$ thick. All experimental processes were conducted under sterile conditions.

\section{Surgical Procedure}

Thirty-five Japanese white rabbits weighing 3.5 to $4.2 \mathrm{~kg}$ were used. All animal procedures were conducted in accordance with the "Guide for the Care and Use of Laboratory Animals" (http:// www.nap.edu/catalog/5140.html). All studies were approved by the Animal Research Committee of Kyoto University School of Medicine.

After intravenous injection of sodium pentobarbital (30 mg/ $/ \mathrm{kg})$, oral intubation was performed, and the animals received mechanical ventilation (model 683; Harvard Apparatus, South Natick, Mass) at a tidal volume of $15 \mathrm{~mL}$ and a minute ventilation rate of 80. General anesthesia was maintained with $1.5 \%$ to $2.0 \%$ isoflurane mixed with oxygen. The heart and omentum were exposed through a median sternotomy and laparotomy. The major branch of the circumflex artery was permanently ligated with 4-0 polypropylene suture to create acute myocardial infarction (MI). Lidocaine $(1 \mathrm{mg} / \mathrm{kg}$ ) was administered intravenously before the completion of this procedure. Careful inspection was maintained for $10 \mathrm{~min}$ utes after the ligation. Ischemia was confirmed by blanching downstream of the ligation and by persistent ST elevation on the electrocardiogram. The omentum was taken out from the peritoneal space into the mediastinum, preserving the arch structure of the GEA. The animals were divided into four groups that received different types of adjunct therapy as follows: group $\mathrm{N}(\mathrm{n}=7$, control) received no additional treatment after creation of the MI; in group $\mathrm{G}(\mathrm{n}=8)$ the omentum including the GEA was wrapped around the infarcted area; in group $F(n=10)$ a gelatin hydrogel sheet incorporating $100 \mu \mathrm{g}$ bFGF was placed over the epicardium of the infarcted area; and in group FG $(\mathrm{n}=10)$ a gelatin hydrogel sheet incorporating $100 \mu \mathrm{g}$ bFGF was placed over the epicardium of the infarcted area, followed by omental wrapping (combined therapy). The bFGF-impregnated hydrogel sheets were fixed to the surface of the heart by stitching with 5-0 polypropylene suture. Before the incision was closed with discontinuous tissue layers, residual air was evacuated from the thorax through silicone tubing attached to a 10-mL syringe. All rabbits were awake within 1 hour after the operation and were kept in an air-conditioned room that was cleaned daily. The rabbits were given free access to water and food thereafter. All animal handling and experiments were conducted in a gentle manner to minimize stress and discomfort to the rabbits, in compliance with the "Guidelines for Use of Laboratory Animals at Kyoto University."

\section{Echocardiographic Measurements}

A commercially available echocardiograph (model SSH-260A U1trasound System; Toshiba Medical, Inc, Tokyo, Japan) including a 7.5-MHz pediatric transducer was used for all studies. Echocardiographic studies were performed before the operation and at 2 and 4 weeks of follow-up. All studies were done with the animals under light anesthesia with intravenous sodium pentobarbital injection under spontaneous respiration. Global ventricular function was estimated by calculating the fractional area change (FAC) of the left ventricle (LV). Two-dimensional M-mode midventricular 

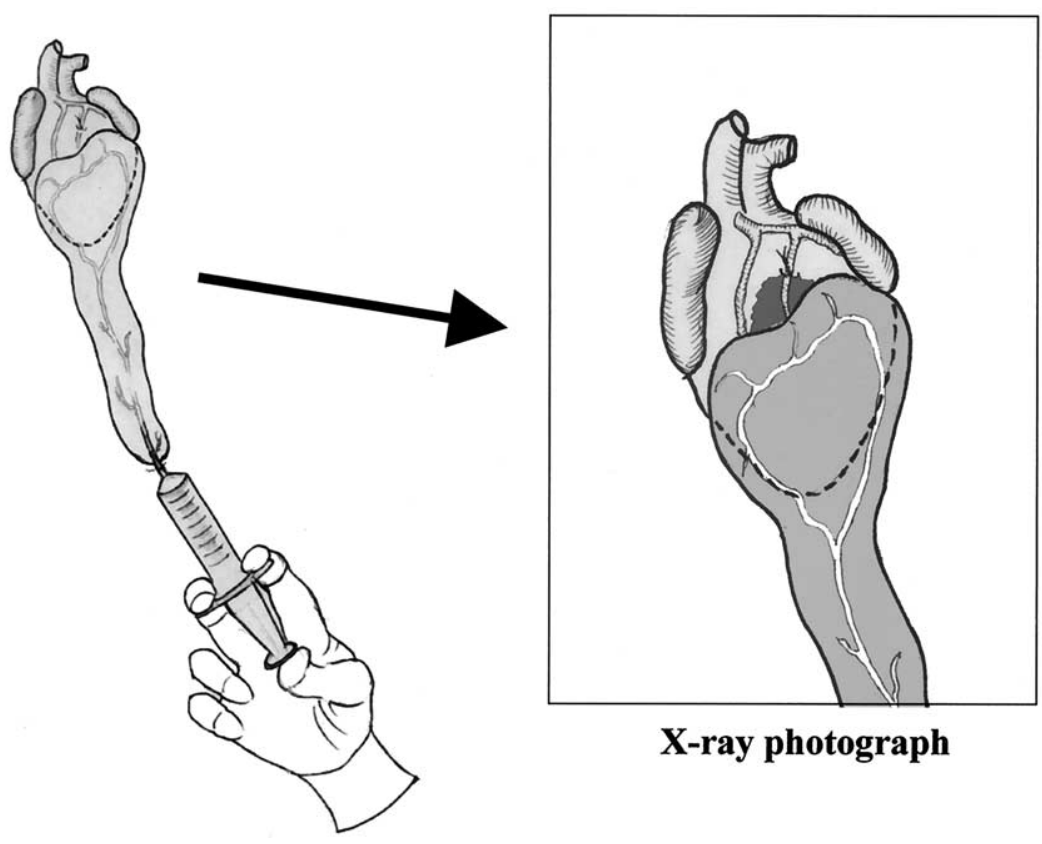

Figure 1. Schema of postmortem angiography. Contrast medium is injected into GEA through small cannula manually inserted through celiac artery. X-ray photograph is taken as in illustration (right).

short-axis images were obtained through a right parasternal approach to determine the FAC by tracing frame by frame throughout the entire cardiac cycle. For each measurement, three consecutive cardiac cycles were averaged. All analyses were done by another observer (K.D.), who was blinded to treatment groups.

\section{Postmortem Angiography}

Communication between the coronary arteries and branches of the GEA in the omentum was evaluated by postmortem angiography at 4 weeks after the operation in groups $\mathrm{G}$ and FG. After intubation, relaparotomy was performed, taking care to avoid injuring the omentum. A 24-mm venous catheter was inserted through the celiac artery, which forms the root of the GEA, and fixed. The chest was reopened to expose the heart wrapped with the omentum. The rabbits were killed with an overdose of pentobarbital, and then the heart and omentum were removed en bloc. A $20-\mathrm{mL}$ portion of heparinized physiologic sodium solution was flushed through the venous catheter into the GEA.

Angiography was performed with an X-ray angiography system (DFW-10B; Toshiba Medical). Angiograms were taken four times every 3 seconds after the start of manual injection of nonionic contrast medium for later analysis (Figure 1). A 3-mL portion of contrast medium was injected by hand for 5 seconds in all cases. Quantitative angiographic analysis of collateral vessel development was performed with a grid overlay composed of $2.5-\mathrm{mm}$ diameter circles arranged at random in the LV distant from the GEA itself. The total number of grid intersections in the LV area, as well as the total number of intersections crossed by a contrastopacified artery, were counted in a single-blind manner. An angiographic score was calculated for each film as the ratio of grid intersections crossed by opacified arteries divided by the total number of grid intersections in the LV area.
Histologic Analysis of MI Area and Arteriolar Density After the angiography, the heart was cut into 2-mm thick slices from apex to base in groups $\mathrm{G}$ and $\mathrm{FG}$. In groups $\mathrm{N}$ and $\mathrm{F}$, the same procedure was performed after the animals had been killed. The slices were immersed in buffered triphenyltetrazolium chloride (TTC) solution and then photographed, and infarct size was calculated with image analysis software (NIH Image 1.61; National Institutes of Health, Bethesda, Md). Each image was analyzed five times and averaged. Infarct size was defined as the percentage of the infarction area within the total area of the LV in each histologic section. As dimensional analysis of the infarction area, three equally spaced measurements of infarct wall from same slices were performed to determine average wall thickness. At the same time, endocardial circumferential length of LV and the scar were measured. For both infarct size and dimensional measurements, the observer was blinded to treatment group. The midcavity area, not used for TTC immersion, was fixed in $4 \%$ paraformaldehyde, embedded in paraffin, and cut into sections $5 \mu \mathrm{m}$ thick. The sections were stained with Masson trichrome to evaluate collagen deposition in comparison with TTC staining.

Other midcavity sections fixed in $4 \%$ paraformaldehyde were stained with a monoclonal antibody against $\alpha$-smooth muscle actin (Sigma, St Louis, Mo), followed by incubation with antimouse immunoglobulin $\mathrm{G}$ (whole molecule). Tetramethylrhodamine isothiocyanate-conjugated secondary antibody (Sigma) was used to detect $\alpha$-smooth muscle cells of arterioles. The sections were counterstained with hematoxylin, eosin, or both. The numbers of arterioles were counted at the infarction border zone under fluorescence microscopy $(\times 100)$ to determine the arteriolar density. Five fields from one section were randomly selected for the arteriole counts. An arteriole was defined as a vessel with a diameter of 20 to $100 \mu \mathrm{m}$. Quantification was performed in a 


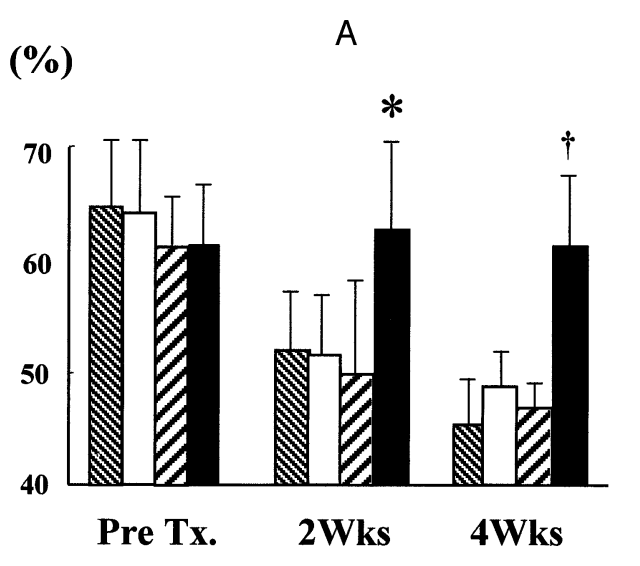

$\left(\mathbf{m m}^{2}\right)$

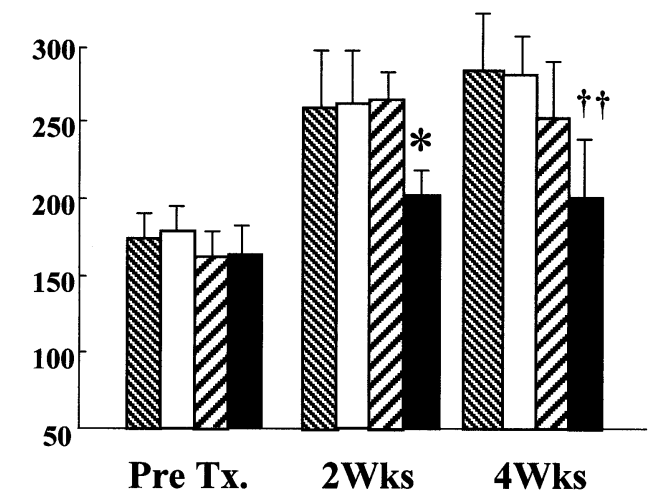

$\mathbb{N}$ Group N $\square$ Group G $\square$ Group F $\square$ Group FG

Figure 2. FAC (A) and LVEDA (B) assessed by echocardiography before operation and at 2 and 4 weeks' follow-up. Pre $T x$, Before treatment. Asterisk indicates $P<.05$ versus groups N, G, and F; dagger indicates $P<.001$ versus groups $N$, G, and $F$; two daggers indicate $P<.01$ versus groups $N, G$, and $F$.

blinded manner with a minimum of three sections for each animal. All animals were used for the analysis.

\section{Statistical Analysis}

Results are expressed as mean $\pm \mathrm{SD}$. Comparisons of echocardiography data among the groups were performed by 2-way analysis of variance (ANOVA). Comparisons of other data among the groups were conducted by 1-way factorial ANOVA. If significance was found for a group, a time effect, or a group-by-time interaction, differences between groups at the same point in time were assessed with 1-way ANOVA followed by post hoc comparisons, when appropriate, with the Student-Newman-Keuls method. Statistical analyses were performed with Statview for Windows version 5.0 (SAS Institute Inc, Cary, NC).

\section{Results}

\section{Global Cardiac Function Assessed by}

Echocardiography

Figure 2 shows the changes in FAC and LV end-diastolic area (LVEDA). None of the FAC and LVEDA values in any of the groups were different before the operation. Two-way ANOVA for FAC showed strong group and time effects and a high group-by-time interaction (respectively $F=9.4, P<$ $.0001, F=20.0, P<.0001$, and $F=4.1, P=.0014)$.

At 2 weeks after the procedure, FAC values in group FG were significantly higher than in groups $\mathrm{N}, \mathrm{G}$, and $\mathrm{F}(P=$ $.03, P=.04$, and $P=.02$, respectively). At 4 weeks, group FG showed higher FAC values than did the other three groups (group FG $65 \pm 7$, group N $46 \pm 1, P=.0004$, group G $51 \pm 7 ; P=.002$, and group F $46 \pm 4, P=.0005$ ).

The changes in LVEDA values, which indicate the loading conditions of LV, showed a pattern similar to that of FAC. At 4 weeks, LVEDA was significantly lower in group
FG than in groups $\mathrm{N}$ and $\mathrm{G}$. No difference reached significance, but LVEDA in group FG had a tendency to be low compared with group F (group FG $198 \pm 45 \mathrm{~mm}^{2}$, group N $280 \pm 50 \mathrm{~mm}^{2} ; P<.001$, group $\mathrm{G} 275 \pm 35 \mathrm{~mm}^{2}, P=$ .003 , and group F $250 \pm 52 \mathrm{~mm}^{2}, P=.06$ ).

Communication Between Coronary Arteries and the GEA in Terms of Angiographic Assessment and Angiographic Score

Angiography was performed in 7 animals in each of groups FG and G. Communication from the GEA was created to the coronary artery though rich collaterals in all 7 animals of group FG. On the other hand, the circumflex artery was identified through poor collaterals in 2 of 7 animals of group G. Representative angiograms recorded from groups FG and $\mathrm{G}$ are shown in Figure 3, $A$. The angiographic score in group FG $(0.82 \pm 0.13)$ was significantly $(P<.001)$ higher than that in group $\mathrm{G}(0.1 \pm 0.11$; Figure $3, B)$.

\section{Infarct Size and Dimensional Analysis of MI}

MI was recognized at the anterolateral wall of the LV, posterolateral wall of the $\mathrm{LV}$, or both in all groups, although there was difference in the infarct size. The infarct ratios determined by analysis of TTC staining in groups N, G, and F were $23 \% \pm 2 \%, 19 \pm 7 \%$, and $16 \% \pm 5 \%$, respectively, which were significantly higher than that in group FG $(10 \%$ $\pm 3 \%)(P<.001, P=.001$, and $P=.04$ for each of the groups; Figure 4). There was no difference between groups $\mathrm{G}$ and F. MI size was reduced to a greater extent in group $\mathrm{F}$ than in group $\mathrm{N}(P=.03)$. Although there was no significant difference in average wall thickness in all 


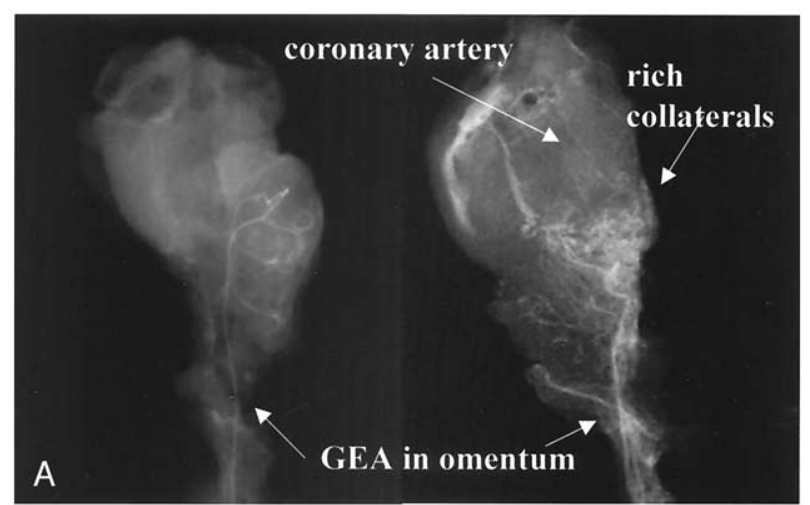

Group G

Group FG

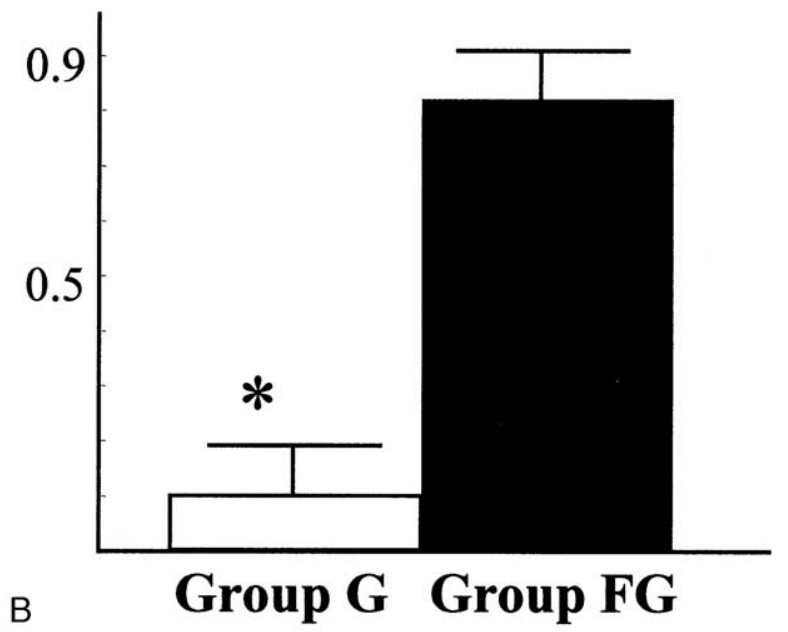

Figure 3. A, Representative postmortem angiogram in groups with omental wrapping (group G) and omental wrapping with bFGF (group FG). Left, in group G, GEA in omentum is opacified, but no communication to coronary arteries is identified. Right, in group FG, numerous collaterals are created between GEA and occluded coronary arteries. B, Angiographic score calculated by postmortem angiographic analysis in groups with omental wrapping (group G) and omental wrapping with bFGF (group FG). Asterisk indicates $\boldsymbol{P}<.001$ versus group FG.

groups, circumferential lengths of LV and the scar were lower in group FG than the other three groups (Table 1).

\section{Immunohistochemical Analysis of New Vessel Formation}

Immunohistochemical staining for anti- $\alpha$-smooth muscle actin revealed that the number of arterioles was increased in the following order: group FG $(23 \pm 5)$ more than group $\mathrm{F}$ $(14 \pm 3)$ more than group $\mathrm{G}(10 \pm 1)$ more than group $\mathrm{N}(4$ \pm 2 ), with each of the differences being significant (Figure $5, A)$. Figure $5, B$, shows representative photomicrographs (original magnification $\times 100$ ) for groups $\mathrm{G}$ and $\mathrm{FG}$.

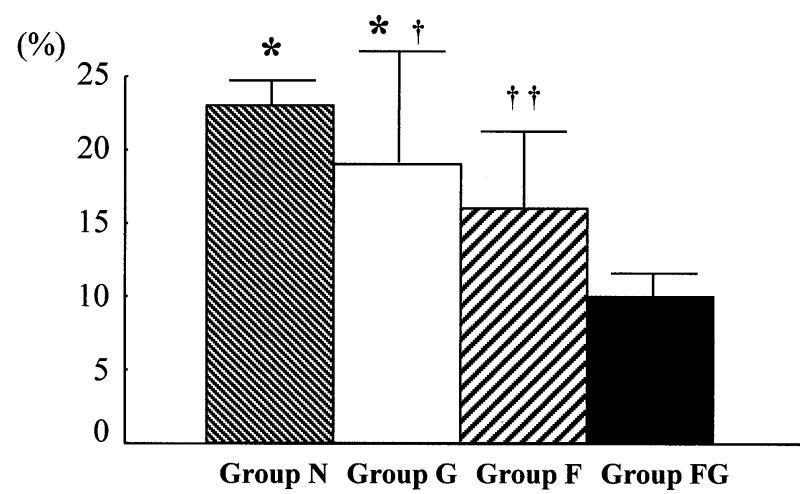

Figure 4. Infarction ratios ( $\%$ of whole $L V$ ) determined by analysis of TTC staining. Asterisk indicates $P<.01$ versus group FG; dagger indicates $P<.05$ versus group $\mathrm{F} ;$ two daggers indicate $P<.05$ versus group FG.

TABLE 1. Dimensional analysis of the scar

\begin{tabular}{lccc}
\hline & $\begin{array}{c}\text { LV circumferential } \\
\text { length (mm) }\end{array}$ & $\begin{array}{c}\text { Circumferential length } \\
\text { of scar }(\mathbf{m m})\end{array}$ & $\begin{array}{c}\text { Infarct wall } \\
\text { thickness (mm) }\end{array}$ \\
\hline Group N & $61 \pm 8$ & $27 \pm 4$ & $1.9 \pm 0.4$ \\
Group G & $58 \pm 5$ & $28 \pm 5$ & $2.1 \pm 0.7$ \\
Group F & $56 \pm 8^{*}$ & $24 \pm 4$ & $2.0 \pm 0.2$ \\
Group FG & $48 \pm 3 \dagger$ & $16 \pm 2 \ddagger$ & $2.5 \pm 0.4$ \\
\hline
\end{tabular}

${ }^{*} P<.005$ versus group $\mathrm{FG}$.

$\dagger P<.001$ versus groups $\mathrm{N}$ and $\mathrm{F}$.

$\ddagger P<.001$ versus groups $\mathrm{N}, \mathrm{G}$, and $\mathrm{F}$.

\section{Discussion}

Multiple experimental and clinical studies have obtained promising results with therapeutic angiogenesis as an alternative to conventional percutaneous coronary intervention or CABG for coronary artery disease that otherwise would have no treatment options. ${ }^{2-9}$ If, however, the donor coronary artery was too far from the target area, too tiny, or diseased or stenosed, conventional therapeutic angiogenesis might not be sufficiently efficacious. Therefore we have proposed a revival of the classic revascularization technique of omentopexy by adding a modern biotechnologic tool, slow release of an angiogenic factor, as an optional strategy for therapeutic angiogenesis.

In this study, the combined therapy involving omentopexy and bFGF administration (group FG) preserved cardiac function better than was seen in the other groups, not only by reducing MI size but also by increasing the number of arterioles. Although myocardial blood flow and hemodynamics were not measured, objective data including postmortem angiography suggested that numerous functional neovessels were connected to the coronary artery from the donor artery. However, the true mechanism of this efficacy was not clear. The pathogenesis of MI, which can be acutely completed within 24 hours, could not be altered by angio- 


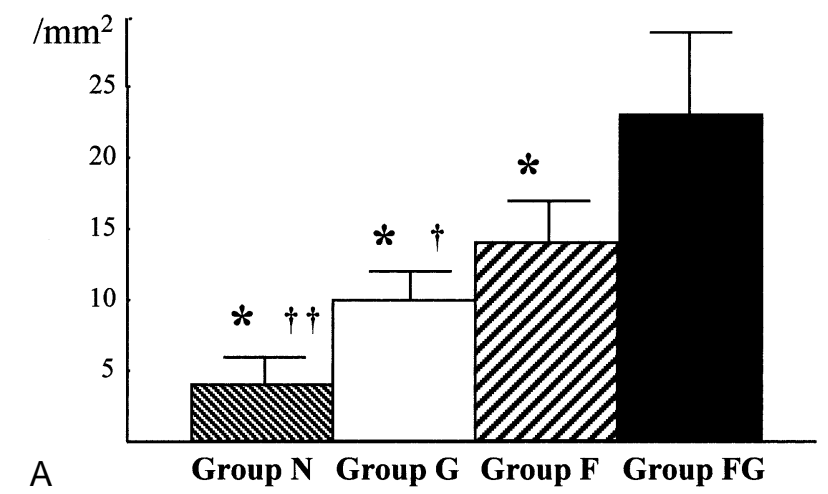

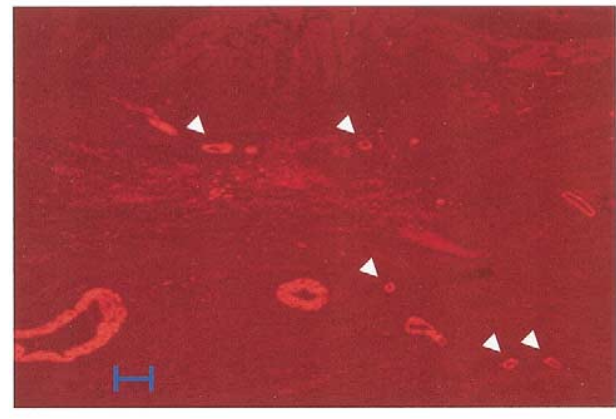

Group G

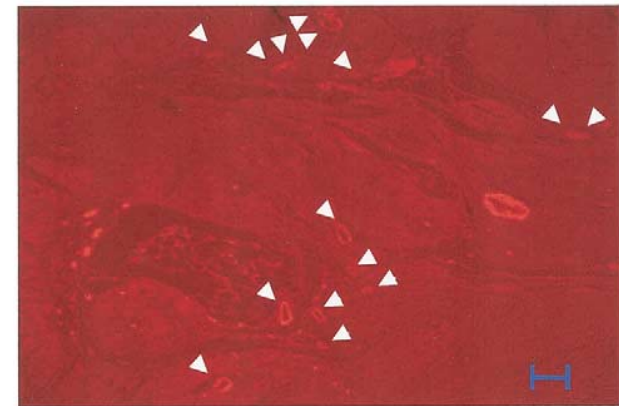

Group FG

Figure 5. A, Number of arterioles counted by immunohistochemical staining. Arteriole was defined as vessel with diameter of 20 to $100 \mu \mathrm{m}$. Asterisk indicates $P<.001$ versus group FG; two daggers indicate $P<.01$ versus group G; dagger indicates $P<.05$ versus group $F$. B, Representative immunohistochemical staining photos (original magnification $\times 100$ ) in groups with omental wrapping (group G) and omental wrapping with bFGF (group FG). Arterioles in border zone of $\mathrm{MI}$ are stained by anti- $\alpha$-smooth muscle actin. White arrows indicate arterioles. Blue bar at lower right is scale of $100 \mu \mathrm{m}$.

genesis. It is possible that the Bio-CABG altered the remodeling process of scar tissue clearance in this study. Preservation of better cardiac function and reduction in MI size may be related to differences in the size of the ventricle caused by altering the remodeling process. The results of dimensional analysis of scar and difference of loading condition were consistent with this presumption.

Omentum including the GEA has classically been used for surgical treatment of not only ischemic heart disease but also complex cardiothoracic problems ${ }^{21,22}$ because the omentum possesses a rich blood and lymph supply and has the additional benefit of producing an angiogenic factor, vascular endothelial growth factor. ${ }^{23}$ In addition to this unique functional character, an omental flap has the advantage of being able to reach and wrap a target organ entirely because of its intrinsic structure. The GEA has become firmly established as an in situ graft conduit with low morbidity, including abdominal complications. ${ }^{24}$ The weak point of omentopexy for myocardial revascularization is its slow and insufficient angiogenesis, similar to that of the
Vineberg operation. In this study, we added a bFGF-containing gelatin sheet to the omental wrapping, and this was found to accelerate angiogenesis effectively relative to omentopexy alone.

Previous studies have shown that gene encoding, virus mediation, and direct injection into the coronary artery or intrapericardially are useful mechanisms for delivering an angiogenic factor to its target. ${ }^{2-7}$ The method used for delivering angiogenic protein in this study was technically simpler and more reliable, and, because it did not involve genes or viruses, it would also be less likely to have adverse effects on a patient. We have reported that prevascularization with bFGF-containing microspheres enhanced the benefits of cardiomyocyte transplantation without increasing the serum level of bFGF (no systemic effects). ${ }^{19}$ Although the technique of delivering bFGF in this study, gelatin sheets attached to the epicardium, differed from injection of beads into the myocardium, the sheets were made of the same material as the beads, and the rate of bFGF release was almost the same. We therefore consider this delivery 
method with gelatin hydrogel to be potentially excellent and widely applicable to other growth factors.

In a clinical setting, Bio-CABG will probably be applicable for treatment of diffuse coronary artery disease that cannot be treated by percutaneous coronary intervention or conventional CABG. This type of coronary artery disease is often recognized in patients receiving hemodialysis or those with severe diabetes mellitus. Even in the era of drugeluting stents, tiny coronary arteries remain a difficult target for interventional catheters. Therefore Bio-CABG has the potential to be a promising new avenue for so-called no option patients with severe coronary disease.

This study was limited as a means of assessing therapeutic angiogenesis in the sense that the acute MI model differs from the clinical situation. Furthermore, myocardial blood flow and hemodynamic effects were not assessed. Angiography and histologic examination do not establish evidence of increased perfusion. Therefore in a future study we are planning to try to estimate myocardial blood flow and the practical efficacy of Bio-CABG directly with the chronic ischemia model in a large animal. Another limitation is that the method for delivering bFGF in this study may not be as good as our previous technique of implanting biodegradable capsules or other techniques, ${ }^{8,9}$ because the attachment of the gelatin sheet to the epicardium may not provide sufficient angiogenic stimuli to the mid or endocardial region of the LV. The omental wrapping may not provide long-term perfusion of the myocardium, and short-term benefits would be anticipated with this procedure. A small MI area was produced in this study even in the control animals. This finding may be related to the use of TTC staining for a short time, which may reduce the accuracy of infarct identification.

In conclusion, we have demonstrated that Bio-CABG can be used to create marked communication between tiny coronary arteries and an extracardiac artery by administration of slow-release bFGF, without surgical anastomosis. This new concept, in which modern biotechnology has been used to revive a classic operation, has the potential to offer revascularization for patients with coronary artery disease who have no other treatment options.

\section{References}

1. Lytle BW, Blackstone EH, Loop FD, Houghtaling PL, Arnold JH, Akhrass R, et al. Two internal thoracic artery grafts are better than one. J Thorac Cardiovasc Surg. 1999;117:855-72.

2. Rajanayagam MA, Shou M, Thirumurti V, Lazarous DF, Quyyumi AA, Goncalves L, et al. Intracoronary basic fibroblast growth factor enhances myocardial collateral perfusion in dogs. $J$ Am Coll Cardiol. 2000;35:519-26.

3. Shou M, Thirumurti V, Rajanayagam S, Lazarous DF, Hodge E, Stiber JA, et al. Effect of basic fibroblast growth factor on myocardial angiogenesis in dogs with mature collateral vessels. J Am Coll Cardiol. 1997;29:1102-6

4. Lopez JJ, Laham RJ, Stamler A, Pearlman JD, Bunting S, Kaplan A, et al. VEGF administration in chronic myocardial ischemia in pigs. Cardiovasc Res. 1998;40:272-81.

5. Banai S, Jaklitsch MT, Shou M, Lazarous DF, Scheinowitz M, Biro S, et al. Angiogenic-induced enhancement of collateral blood flow to ischemic myocardium by vascular endothelial growth factor in dogs. Circulation. 1994;89:2183-9.

6. Tio RA, Tkebuchava T, Scheuermann TH, Lebherz C, Magner M, Kearny $\mathrm{M}$, et al. Intramyocardial gene therapy with naked DNA encoding vascular endothelial growth factor improves collateral flow to ischemic myocardium. Hum Gene Ther. 1999;10:2953-60.

7. Mack CA, Patel SR, Schwarz EA, Zanzonico P, Hahn RT, Ilercil A, et al. Biologic bypass with the use of adenovirus-mediated gene transfer of the complementary deoxyribonucleic acid for vascular endothelial growth factor 121 improves myocardial perfusion and function in the ischemic porcine heart. $J$ Thorac Cardiovasc Surg. 1998;115:168-76.

8. Laham RJ, Sellke FW, Edelman ER, Pearlman JD, Ware JA, Brown DL, et al. Local perivascular delivery of basic fibroblast growth factor in patients undergoing coronary bypass surgery: results of a phase I randomized, double-blind, placebo-controlled trial. Circulation. 1999; 100:1865-71.

9. Ruel M, Laham RJ, Parker JA, Post MJ, Ware JA, Simons M, et al. Long-term effects of surgical angiogenic therapy with fibroblast growth factor 2 protein. J Thorac Cardiovasc Surg. 2002;124:28-34.

10. O'Shaughnessy L. Surgical treatment of cardiac ischemia. Lancet. 1937;232:185-94.

11. Vineberg AM, Miller G. Internal mammary coronary anastomoses in surgical treatment of coronary artery insufficiency. Can Med Assoc J. 1951;64:204-10

12. Vineberg AM. Revascularization of the entire heart by internal mammary artery implantation, epicardiectomy and free omental graft. Can Med Assoc J. 1966;94:378-85.

13. Vineberg AM, Kato Y, Pirozynski WJ. Experimental revascularization of the entire heart. Evaluation of epicardiectomy, omental graft, and/or implantation of the internal mammary artery in preventing myocardial necrosis and death of the animal. Am Heart J. 1966;72:79-93.

14. Friedbacker K. Omentum as source of nutrition to experimentally produced myocardial ischemia. Surg Gynecol Obstet. 1942;75:110-3.

15. Vineberg A. Revascularization of the right and left coronary arterial systems. Internal mammary artery implantation, epicardiectomy and free omental graft operation. Am J Cardiol. 1967;19:344-53.

16. Streider JW, Clute HM, Graubiel A. Cardioomentopexy in the treatment of angina pectoris. $N$ Engl J Med. 1940;222:41-3.

17. Iwakura A, Tabata Y, Miyao M, Ozeki M, Tamura N, Ikai A, et al. Novel method to enhance sternal healing after harvesting bilateral internal thoracic arteries with use of basic fibroblast growth factor. Circulation. 2000;102(19 Suppl 3):III307-11.

18. Iwakura A, Tabata Y, Tamura N, Doi K, Nishimura K, Nakamura T, et al. Gelatin sheet incorporating basic fibroblast growth factor enhances healing of devascularized sternum in diabetic rats. Circulation. 2001;104(12 Suppl 1):I325-9.

19. Sakakibara Y, Nishimura K, Tambara K, Yamamoto M, Lu F, Tabata $\mathrm{Y}$, et al. Prevascularization with gelatin microspheres containing basic fibroblast growth factor enhances the benefits of cardiomyocyte transplantation. J Thorac Cardiovasc Surg. 2002;124:50-6.

20. Tabata Y, Nagano A, Ikada Y. Biodegradation of hydrogel carrier incorporating fibroblast growth factor. Tissue Eng. 1999;5:127-38.

21. Shrager JB, Wain JC, Wright CD, Donahue DM, Vlahakes GJ, Moncure AC, et al. Omentum is highly effective in the management of complex cardiothoracic surgical problems. J Thorac Cardiovasc Surg. 2003;125:526-32.

22. Levashev YN, Akopov AL, Mosin IV. The possibilities of greater omentum usage in thoracic surgery. Eur J Cardiothorac Surg. 1999; $15: 465-8$.

23. Zhang QX, Magovern CJ, Mack CA, Budenbender KT, Ko W, Rosengart TK. Vascular endothelial growth factor is the major angiogenic factor in omentum: mechanism of the omentum-mediated angiogenesis. J Surg Res. 1997;67:147-54.

24. Suma H, Amano A, Horii T, Kigawa I, Fukuda S, Wanibuchi Y. 
Gastroepiploic artery graft in 400 patients. Eur J Cardiothorac Surg.. 1996;10:6-10.

\section{Discussion}

Dr Hiroyuki Tsukui (Pittsburgh, Pa). I have two questions. First, how long does the bFGF effect last? Second, what is an appropriate volume? Do you use $100 \mu \mathrm{g}$ ?

Dr Ueyama. We use the administration method of bFGF by gelatin hydrogel sheet. We used that dose of, as you say, $100 \mu \mathrm{g}$ bFGF only in this study. But in another study we have already reported that a $100-\mu \mathrm{g}$ dose of bFGF was enough to inducing angiogenesis for another target organ.

Dr Tsukui. So do you mean that a flow rate of $100 \mu \mathrm{g}$ is appropriate? Or if you used it 12 or more weeks at $200 \mu \mathrm{g}$ or another dose, would that be better for revascularization?

Dr Ueyama. I don't have an exact answer to your question now, but I'd guess that one would not need to use more than 100 for FGF for better effect.

Dr Andrew S. Wechsler (Philadelphia, $P a$ ). Did you happen to look and see how deep in the myocardium the new blood vessels went? Did they reach the subendocardial surface or the mid myocardium, or was it primarily an epicardial blush?

Dr Ueyama. I think the results of angiography suggested that the new vessel from the GEA passed through the epicardium and myocardium and bypassed to the coronary artery.

Dr Virna Sales (Boston, Mass). Would you be able to see a prospective microencapsulating this bFGF with a hydrogel and have that kind of delivery?

Dr Ueyama. Yes, we did. The material is the same that we used in this study.

Dr Sales. But would that be possible to microencapsulate this?

Dr Ueyama. In another study we used microspheres including bFGF injected into myocardium. Many angiogenesis occurred in the myocardium between each coronary artery but not from an extracardiac donor artery.

Dr Sales. What is the degradation time of this hydrogel? You said it was biodegradable.

Dr Ueyama. It takes about 7 to 10 days for complete degradation. 